\title{
Speed and accuracy of same and different responses in perceptual matching
}

\author{
ROGER RATCLIFF \\ Yale University, New Haven, Connecticut 06520 \\ and \\ MICHAEL J. HACKER \\ Harvard University, Cambridge, Massachusetts 02138
}

\begin{abstract}
Models of the visual perceptual matching process are evaluated in two experiments in which the relative speeds of "same" and "different" responses were manipulated. In the first experiment, subjects were tested under two bias conditions: they were instructed to respond "same" only when sure or to respond "different" only when sure. Such bias is found to have a very large effect on the speed and accuracy of "same" and "different" responses, changing "same" responses from being faster than "different" responses (the usual result) in the sure "different" condition to being slower in the sure "same" condition. In the second experiment, the relative speed of "same" responses was slowed significantly by the addition of difficult "different" judgments. These results are used to argue against models that contain an identity matcher and against models that have separate components to account for errors and reaction time. We also point out that, although it is well known that the relative speeds of positive and negative responses are subject to bias manipulations, this fact has been ignored by many researchers in developing models in which the reaction time difference between positive and negative responses is used as a measure of a stage of processing.
\end{abstract}

Several models of the visual perceptual matching task use the difference in reaction time between "same" and "different" responses to measure the duration of a stage of processing (Bamber, 1969; Proctor, 1981; Taylor, 1976). For example, Bamber (1969) has developed a serial processing model to account for results from the perceptual matching task. He found that the serial processing model could be adequately fit to reaction time for "different" responses but that "same" responses were significantly faster than predicted. This was taken as evidence for an identity matching process such that a "different" response could only result from the slow serial processor but a "same" response could result from either the serial processor or the fast identity matcher. Likewise, Taylor (1976) has examined serial and parallel processing models, and, although he favored a parallel processing model over a serial processing model, he was still obliged to include an identity

This research was partially supported by NSF Grant BNS 7921028 to W. K. Estes and NICHD Grant HD 13318 to Gail McKoon and Roger Ratcliff. Michael Hacker was supported by NIMH Training Grant 15125. This paper was prepared while Roger Ratcliff was a visiting assistant professor at Rockefeller University, enjoying the hospitality and stimulation of W. K. Estes' Lab. We wish to thank Gail McKoon for her comments on this paper. Requests for reprints should be sent to Roger Ratcliff, Department of Psychology, Yale University, Box 11A Yale Station, New Haven, Connecticut 06520 . matching process. Finally, Proctor (1981) has compared the same match-physical match reaction time difference with the same-different reaction time difference and has concluded that, because these differences are equal, it could be assumed that the same process underlies performance on both tasks.

It is our aim to demonstrate that the same-different difference is highly dependent upon the subject's bias setting and that this difference can be easily manipulated to produce any pattern of reaction time differences. We will argue that such a demonstration shows that models based on the same-different difference are misguided. We will also describe models that are capable of dealing with these data without recourse to a fast identity matcher. Before describing the experiments, we shall discuss recent attempts to model bias in experimental situations in which the dependent measure is detection rate and in situations in which the dependent measure is reaction time.

It is easy to show that subjects can change their biases in tasks such as detection and recognition. For example, in a task requiring detection of a signal in noise, subjects can adjust their biases along a continuum from responding "yes" only when very sure of the signal's presence to responding "no" only when very sure of signal's absence. This bias can be separated from the subject's ability to discriminate between signal and noise with a quantitative model (Green \& Swets, 1966). 
Another bias that has come under scrutiny lately has been speed-accuracy bias. Subjects can alter their speed-accuracy bias from being fast at the cost of accuracy to being accurate at the cost of speed (see Pachella, 1974). The problems posed by speed-accuracy bias for the interpretation of reaction time experiments can be illustrated by two recognition memory experiments reported by Ratcliff (1978). In one experiment, rate of presentation of study items was a between-sessions variable. It was found that, as study time per item increased, accuracy improved but (counter to intuitions) reaction time also increased (the better the memory is, the faster and more accurate the responses should be). In a second experiment, study time per item was made a within-studylist variable (some items were on for short times and some for longer times). In this case, subjects could not adjust their speed-accuracy criteria before retrieving information about a test item. It was found that, as study time per item increased, accuracy improved, but now reaction time decreased. It was argued that the increase in reaction time in the betweensessions design was the result of an adjustment in speed-accuracy bias. From this example, we can see that there are difficult methodological problems involved in experiments that allow the subject to adjust speed-accuracy bias as a function of condition, because observed reaction time differences may simply be the result of bias rather than a change in the processes hypothesized for the experiment. On the other hand, the ability of subjects to change their speed-accuracy bias is an important property of the processing system, and models of processing should attempt to account for the flexibility that the subject has in setting speed-accuracy bias.

The bias we will consider in this paper is yes-no bias in reaction time experiments. This bias is important not only in the perceptual matching task, but also in other tasks that use reaction time as the dependent measure. Differences in yes-no reaction time have been used as absolute measures of the durations of processing stages (e.g., Anderson, 1976, p. 268; Carpenter \& Just, 1975; Clark \& Chase, 1972; Meyer, 1970; Murdock, 1974, p. 273; Singer, 1981) and as measures of the relative durations of nondecision processes (Anderson \& Bower, 1973; Atkinson \& Juola, 1973; Schneider \& Shiffrin, 1977; Smith, Shoben, \& Rips, 1974; Sternberg, 1969). Although the experiments presented below do not directly address all the areas of research represented in these references, the idea that the relative speeds of "yes" and "no" responses are manipulable has important implications for these areas.

The attribution of the absolute difference between positive and negative response times to the duration of a specific processing stage is particularly perilous if it can be shown that the difference is easily manip- ulated and covaries with accuracy. If the difference can actually be reversed, it is either necessary to reject the hypothesized stage altogether or, almost certainly, to concede that the positive-negative response time difference is at best a confounded measure and to look for a more comprehensive account of both speed and accuracy.

A large body of evidence demonstrating the differential effects of stimulus frequency, payoffs, expectancies, and other bias-related manipulations upon the speed and accuracy of simple forced-choice responses has been gathered over the years (e.g., Colthart \& Curthoys, 1968; Laming, 1968; Link, 1975; Pachella, 1974; Sternberg, 1969; Theios \& Walter, 1974). Of particular interest is the study of Colthart and Curthoys (1968). They carried out speed-accuracy manipulations in a task in which subjects were required to match tones (the auditory analog of the visual matching task considered here). They were able to show that the relative speed and accuracy of "same" and "different" judgments were manipulable so that when "same" trials were most frequent, "same" responses were faster and more accurate, and when "different" trials were more frequent, "different" responses were faster and more accurate. An explanation of these results was presented in terms of a signal detection model and a sequential sampling model (see also Krueger, 1979, for a quantitative application of such models to the tone matching task). Despite these demonstrations, the relevance of these results seems not to have been fully appreciated in the development and assessment of stage models for more complex yes-no decision tasks.

We shall now present two experiments in which the relative speeds of "same" (positive) and "different" (negative) responses were manipulated (along with error rates) in the visual perceptual matching task.

\section{EXPERIMENT 1}

In typical experiments by Bamber (1969) and Taylor (1976), subjects studied strings of one, two, three, or four letters and were tested with a letter string of the same length. The subjects were required to respond "same" if the letter strings matched in each position and "different" otherwise. In this experiment, we restricted our experimental conditions to letter strings of four-letter length in order to collect more data per condition. The manipulation of major interest was the instruction to the subject either to be cautious on "same" responses or cautious on "different" responses.

\section{Method}

On each trial, subjects were presented with four letters to study for $1 \mathrm{sec}$. There followed a 200 -msec blank, a 100-msec mask 
(SSSS), and a 200-msec blank delay, and then the test stimulus was presented for 100 msec. The screen remained blank until the subject responded, indicating whether the test letter string was the same as the study string. Subjects were given immediate feedback on their accuracy (a plus sign for a correct response and a minus sign for an error). The feedback was displayed for $500 \mathrm{msec}$, and then there was a 1,500 -msec blank delay until the next stimulus was presented. A session consisted of four blocks of 96 test trials, each block preceded by four warm-up trials and followed by a short rest period. The stimulus letters were randomly selected from the set C, D, F, H, J, K, L, R, S, and T, without replacement. This set of letters was chosen to be minimally confusable (Taylor, 1976). On each trial, the test letters were either identical to the study letters and appeared exactly in the same position as the study letters or had one, two, three; or four letters replaced by the remaining letters in the stimulus pool. On half of the trials, the test string matched the study string, and on the other half of the trials, one, two, three, or four letters mismatched equally often. The order of these trials was uniquely randomized for each subject and each session.

There were four subjects: the second author, a Rockefeller University faculty volunteer, and two local long-term subjects who were paid $\$ 3.00$ per 45 -min session. Each subject participated in six experimental sessions preceded by one practice session. Three consecutive experimental sessions per subject constituted the cautious "same" condition. Subjects were instructed to respond "same" only when absolutely sure. The other three experimental sessions constituted the cautious "different" condition in which subjects were to respond "different" only when absolutely sure. Response assignment (dominant hand) was crossed with instruction order.

Stimulus presentation, timing, randomization, and response recording were controlled by a PDP 81 computer. Subjects viewed the stimuli on a CRT screen from a distance of approximately $60 \mathrm{~cm}$. The letters were constructed from a $5 \times 6 \mathrm{dot}$ matrix, and the four test letters subtended an angle of $3.2 \mathrm{deg}$. Responses were made by releasing one of two microswitches (using the index fingers)-one for "same," the other for "different"-and reaction time was recorded to the nearest $2 \mathrm{msec}$.

\section{Results}

The major results are shown in Table 1 . In the cautious "same" condition, "same" responses were slower than all the "different" response conditions except the "one-different" condition ("same," $573 \mathrm{msec}$; average "different," $515 \mathrm{msec}$ ), whereas

Table 1

Accuracy and Correct Reaction Time Results for Experiment 1

\begin{tabular}{|c|c|c|c|c|c|c|}
\hline & \multicolumn{3}{|c|}{ Cautious Same } & \multicolumn{3}{|c|}{ Cautious Different } \\
\hline & $\mathbf{N}$ & $\mathrm{RT}$ & A & $\mathbf{N}$ & RT & $\mathbf{A}$ \\
\hline & \multicolumn{6}{|c|}{ Same } \\
\hline & 2303 & 573 & .891 & 2303 & 472 & .967 \\
\hline & \multicolumn{6}{|c|}{ Different } \\
\hline $\begin{array}{l}1 \\
2 \\
3 \\
4\end{array}$ & $\begin{array}{l}576 \\
576 \\
576 \\
576\end{array}$ & $\begin{array}{l}605 \\
517 \\
480 \\
461\end{array}$ & $\begin{array}{l}.797 \\
.955 \\
.976 \\
.971\end{array}$ & $\begin{array}{l}574 \\
576 \\
575 \\
576\end{array}$ & $\begin{array}{l}695 \\
584 \\
531 \\
518\end{array}$ & $\begin{array}{l}.646 \\
.894 \\
.937 \\
.969\end{array}$ \\
\hline
\end{tabular}

Note $-N=$ number of responses: $R T=$ reaction time in milliseconds; $A=$ accuracy. There was an upper reaction time cut-off of $2 \mathrm{sec}$ and a lower cut-off of $100 \mathrm{msec}$. Standard errors on reaction times were as follows. Same: $573 \pm 4 \mathrm{msec}, 472 \pm$ $3 \mathrm{msec}$; different: $460 \pm 7 \mathrm{msec}, 500 \pm 10 \mathrm{msec}, 600 \pm 14 \mathrm{msec}$, $700 \pm 18 \mathrm{msec}$. in the cautious "different" condition, "same" responses were faster than all "different" conditions ("same," $472 \mathrm{msec}$; average "different," $582 \mathrm{msec}$ ). There were also corresponding changes in accuracy; for the cautious "same" condition, there was a lower probability of an incorrect "same" response and a higher probability of an incorrect "different" response than in the cautious "different" condition.

These results suggest that the same-different reaction time difference is subject to criterion effects in much the same way as are accuracy differences. Further discussion will be delayed until the results of Experiment 2 have been presented.

\section{EXPERIMENT 2}

In this experiment, the same-different reaction time difference was manipulated by the addition of difficult negative test conditions. (There was no speed-accuracy manipulation in this experiment.) As in the preceding experiment, four-letter strings were used, but half the "different" trials involved pairwise transpositions rather than substitutions of letters. In many other experiments (e.g., Bamber, 1969; Taylor, 1976), it has been shown that with negative test strings consisting of substitutions, reaction time to "same" was faster than reaction time to all "different" conditions. It was anticipated that the transposition conditions would be considerably more difficult than the replacement conditions and so may affect the relative speeds of "same" and "different" responses.

\section{Method}

The experiment is similar to Experiment 1 in many respects; only the differences will be described here.

Four local long-term subjects participated in this experiment. Each subject participated in six sessions (the first of which was discarded as a practice session). There were 432 test trials per session, of which 216 were "same" trials and 216 were "different" trials. Of the 216 "different" trials, 108 consisted of 18 replications of the 6 double substitutions (Positions 1-2, 1-3, 1-4, 2-3, 2-4, and 3-4) and 108 consisted of 18 replications of the 6 pairwise transpositions (matched for position against the substitutions). Each block of 144 test trials was preceded by five warmup trials. Timing, feedback, the character set, and presentation conditions were identical to those in Experiment 1.

\section{Results}

The main results of this experiment are shown in Table 2. The first result to note is that reaction time for "same" responses was $90 \mathrm{msec}$ slower than reaction time for responses in the fastest "different" conditions. Second, the transposition conditions show significantly poorer performance than do the substitution conditions. Furthermore, distance effects were found in the transposition data. If adjacent letters were transposed, performance was poorer than if nonadjacent letters were transposed. 
Table 2

Accuracy and Correct Reaction Time Results for Experiment 2

\begin{tabular}{|c|c|c|c|}
\hline & Accuracy & $\begin{array}{l}\text { Reaction Time } \\
\text { (in Milliseconds) }\end{array}$ & $\begin{array}{c}\text { Number of } \\
\text { Observations }\end{array}$ \\
\hline & \multicolumn{3}{|c|}{ Same } \\
\hline & .986 & 536 & 4319 \\
\hline & \multicolumn{3}{|c|}{ Different, Transpositions } \\
\hline $1-2$ & .936 & 550 & 360 \\
\hline $1-3$ & .981 & 508 & 360 \\
\hline 14 & .986 & 488 & 360 \\
\hline $2-3$ & .858 & 648 & 359 \\
\hline $2-4$ & .961 & 532 & 360 \\
\hline \multirow[t]{2}{*}{34} & .642 & 766 & 360 \\
\hline & \multicolumn{3}{|c|}{ Different, Substitutions } \\
\hline $1-2$ & .992 & 452 & 360 \\
\hline $1-3$ & 1.000 & 443 & 360 \\
\hline 14 & 1.000 & 448 & 359 \\
\hline $2-3$ & .997 & 446 & 359 \\
\hline $2-4$ & .994 & 452 & 360 \\
\hline $3-4$ & .981 & 513 & 360 \\
\hline
\end{tabular}

Note-There was an upper reaction time cut-off of $2 \mathrm{sec}$ and a lower cut-off of $100 \mathrm{msec}$. Standard errors on reaction times were as follows. Same: $536 \pm 2.5$ msec; different: $450 \pm 5 \mathrm{msec}$, $500 \pm 7 \mathrm{msec}, 600 \pm 10 \mathrm{msec}, 750 \pm 14 \mathrm{msec}$.

\section{DISCUSSION}

The main aim of the experiments presented above was to investigate the effect of criterion changes on the same-different reaction time difference. In Experiment 1 , this was done by giving explicit instructions to subjects to bias their confidence in "same" and "different" responses. It was found that biasing subjects to be cautious either on "same" responses or on "different" responses produced the changes in accuracy that would be expected from a decision theory (signal detection theory) perspective. Along with these changes in accuracy, it was found that the same-different reaction time difference changed direction. In the usual experiment (without biasing instructions), "same" responses are faster than "different" responses, so the results of the first experiment show that the same-different reaction time difference is in fact manipulable in the same way that accuracy is manipulable. In Experiment 2, a new category of negatives (transpositions), designed to be difficult, was added in order to reduce the "same" advantage. It was found that, in fact, "same" responses were slower than the faster categories of negative responses (double replaces). This result complements the results from Experiment 1 by showing that another manipulation besides instruction will eliminate the "same" reaction time advantage.

These results have important implications for models of the matching task that account for the "same" reaction time advantage by invoking an additional mechanism (e.g., an identity matcherBamber, 1969; Taylor, 1975; or an encoding process-
Proctor, 1981) for "same" and "different" judgments. Such models cannot predict the slow "same" reaction time in the cautious "same" condition of Experiment 1 and Experiment 2. For example, in Bamber's (1969) model, the subject is able to produce a "same" response either from an identity match or from the slower serial process. Neither of these mechanisms would produce "same" responses much more slowly than they would "different" responses. Furthermore, in Bamber's model, errors are the result of loss of information from the visual information store. Thus, the model cannot account for the speedaccuracy changes demonstrated here, except by the manipulation of unrelated mechanisms. The model supported by Taylor (1976) has similar problems, because it also has an identity matcher separate from the parallel comparison mechanism and because errors arise from a guessing mechanism that is independent of the mechanisms that give rise to reaction time effects. In a similar vein, Proctor's model deals only with reaction time and explains the "same" advantage in terms of faster processing of physically identical letters as opposed to physically different letters in the nonmatch condition. Thus, the model cannot deal with the criterion effects obtained in Experiments 1 and 2. Furthermore, Proctor's model is mute on the relationship between accuracy and reaction time and so cannot deal with the combined speed-accuracy criterion effects.

A model that is capable of accounting for some of the effects obtained in Experiments 1 and 2 is that proposed by Krueger (1978). In his model, a letter is composed of a number of features. If a test letter matches a study letter, then it is assumed that there are no nonmatching features; if the test and study strings do not match, then there is a small number of nonmatching features. The comparison process is executed in a number of passes. On each pass, the number of feature nonmatches out of the total number of features is counted: if this number is above one criterion, a "different" response is initiated; if this number is below another criterion, a "same" response is initiated. If the number of nonmatches is between the two criteria, another pass is performed and the number of nonmatches (summed across passes) is again checked against two (adjusted) criteria. Passes continue to be executed for nonterminated processes until a deadline is reached. During the comparison process, a feature match may be misperceived as a feature nonmatch or a feature nonmatch may be misperceived as a feature match. These misperceptions represent noise in the comparison process and have low probability (e.g., less than .1).

Krueger's model is of the class of sequential sampling models and, as such, relates accuracy and reaction time. This model is capable of dealing with the same-different effects presented in Experiments 1 
and 2. To account for the instruction manipulation, it is only necessary to assume that subjects adjust their upper and lower criteria. For example, in the cautious "same" condition, subjects require more feature matches before producing a "same" response than they do in the cautious "different" condition. Similarly, for "different" responses, subjects require more feature nonmatches in the cautious "different" condition than they do in the cautious "same" condition. To account for the slow "same" responses in Experiment 2, it is only necessary to assume that the subject sets a stricter "same" criterion in order to discriminate between "same" trials and the very similar transposition trials. Thus, the substitution "different" trials produce very rapid responses because the "different" criterion is reached quickly (in few passes) relative to the transposition conditions (adjacent transpositions more difficult than nonadjacent transpositions).

Distance effects have been accounted for by a model developed by Ratcliff (1981). This model integrates a diffusion retrieval process (a sequential sampling process) and a model for the representation of the stimuli in memory. The representation of letters in the study and test strings is assumed to be distributed across spatial position, and the matching process is carried out by assessing amount of overlap between the study and test strings. If adjacent letters are transposed, then the amount of overlap is large, almost as large as in the "same" condition. This accounts for the distance effects; other same-different effects are accounted for in the same way as in Krueger's sequential sampling model.

In conclusion, the results of Experiments 1 and 2 show that the same-different reaction time difference is manipulable. These results are inconsistent with models such as Bamber's (1969), Proctor's (1981), and Taylor's (1976) but can be handled quite easily by the sequential sampling models presented by Krueger (1978) and Ratcliff (1981).

\section{REFERENCES}

Andenson, J. R. Language, memory and thought. Hillsdale, N.J: Erlbaum, 1976.

ANDERSon, J. R., \& Bower, G. H. Human associative memory. Washington, D.C: Winston, 1973.

AtKinson, R. C., \& Juola, J. F. Factors influencing speed and accuracy of word recognition. In S. Kornblum (Ed.), Attention and performance IV. New York: Academic Press, 1973.
Bamber, D. Reaction times and error rates for "same".."different" judgments of multidimensional stimuli. Perception \& Psychophysics, 1969, 6, 169-174.

Carpente r, P., \& Just, M. Sentence comprehension: A psycholinguistic processing model of verification. Psychological Review, 1975, 82, 45-73.

Clark, H. H., \& Chase, W. G. On the process of comparing sentences against pictures. Cognitive Psychology, 1972, 3, 472-517.

Colthart, M., \& Curthoys, I. Short-term recognition memory for pitch: Effect of a priori probability on response times and error rates. Perception \& Psychophysics, 1968, 4, 85-89.

Green, D. M., \& Swets, J. A. Signal detection theory and psychophysics. New York: Wiley, 1966.

KrUeger, L. A theory of perceptual matching. Psychological Review, 1978, 85, 278-304.

KrUEger, L. A model of unidimensional perceptual matching. Journal of Experimental Psychology: Human Perception and Performance, 1979, 5, 277-288.

LAMING, D. Information theory of choice reaction time. New York: Wiley, 1968.

LiNK, S. The relative judgment theory of choice reaction time. Journal of Mathematical Psychology, 1975, 12, 114-135.

MEYER, D. E. On the representation and retrieval of stored semantic information. Cognitive Psychology, 1970, 1, 242-300.

Murdock, B. Human memory: Theory and data. Potomac, Md: Erlbaum, 1974.

Pachella, R. G. An interpretation of reaction time in information processing research. In B. Kantowitz (Ed.), Human information processing: Tutorials in performance and cognition. Hillsdale, N.J: Erlbaum, 1974.

Proctor, R. W. A unified theory for matching task phenomena. Psychological Review, 1981, 88, 291-326.

RAtcliff, R. A theory of memory retrieval. Psychological Review, $1978,85,59-108$.

RAtchifF, $\mathbf{R}$. A theory of order relations in perceptual matching. Psychological Review, 1981, 88, 552-572.

SchNeider, W., \& Shiffrin, R. M. Controlled and automatic human information processing. Psychological Review, 1977, 86, $1-66$.

SingER, M. Verifying the assertions and implications of language. Journal of Verbal Learning and Verbal Behavior, 1981, 20, 46-60.

Smith, E. E., Shoben, E. J., \& RIPs, L. J. Structure and process in semantic memory: A featural model for semantic decisions. Psychological Review, 1974, 8, 214-241.

STE RNBERG, S. Memory-scanning: Mental processes revealed by reaction time experiments. American Scientist, 1969, 57, 421-457.

TAYLOR, D. Effect of identity in the multiletter matching task. Journal of Experimental Psychology: Human Perception and Performance, 1976, 2, 417-428.

Theios, J., \& Walter, D. G. Stimulus and response frequency and sequential effects in memory scanning reaction times. Journal of Experimental Psychology, 1974, 102, 1092-1099.

(Manuscript received April 13, 1981; revision accepted for publication June 9, 1981.) 